\title{
NIVEL DE ESTRÉS EN PADRES DE NIÑOS HOSPITALIZADOS EN UNIDADES CRÍTICAS PEDIÁTRICAS Y NEONATALES
}

\author{
STRESS LEVEL IN PARENTS OF HOSPITALIZED CHILDREN IN \\ PEDIATRIC AND NEONATAL CRITICAL CARE UNITS
}

\section{NÍVEL DE ESTRESSE EM PAIS DE CRIANÇAS INTERNADAS EM UNIDADES CRÍTICAS PEDIÁTRICAS E NEONATAIS}

\author{
Ángela Astudillo Araya* \\ Patricia Silva Pacheco** \\ Jenifer Daza Sepúlveda***
}

\begin{abstract}
RESUMEN
Introducción: Enfermedad y hospitalización son eventos críticos que enfrenta un niño, donde la reacción de estrés en toda la familia es común durante la enfermedad o una estancia hospitalaria grave. Objetivo: Determinar el nivel de estrés en padres de niños hospitalizados en las unidades críticas de pediatría y neonatología y su relación con características sociodemográficas del padre y/o niño/a. Material y método: Estudio cuantitativo, descriptivo-correlacional, de corte transversal. Se estudió una muestra de 41 padres, que fueron elegibles y estuvieron presentes durante mayo de 2017 en las unidades críticas de pediatría y neonatología de un hospital ubicado en la Región de Nuble, Chile. Se aplicó un cuestionario de caracterización y la versión en español de la Escala de Estrés Parental adaptada por Polaino-Lorente y Ochoa. Los datos fueron procesados con SPSS 15.0, realizando pruebas t-student y ANOVA de un factor. Resultados: En relación al puntaje global de estrés, 48,8\% clasificó el grado de estrés como extremadamente estresante; 29,3\% muy estresante; $12,2 \%$ moderadamente estresante y los demás poco y nada estresante. El estrés en padres es mayor durante la primera semana de hospitalización del niño, en ámbitos de comunicación del personal sanitario y la alteración del rol de padres, siendo ambas significativas. Conclusión: El estrés en padres con un hijo hospitalizado en unidades críticas resulta en la mayoría de los casos extremo, siendo mayor durante la primera semana de hospitalización del niño.
\end{abstract}

Palabras clave: Estrés psicológico; Padres, Enfermería neonatal; Enfermería pediátrica; Unidades de Cuidados Intensivos.

\footnotetext{
ABSTRACT

Introduction: Illness and hospitalization are critical events for a child. It is common that the whole family experiences a stress reaction while the child is seriously ill or hospitalized. Objective: The purpose of this

*Enfermera. Magíster en Pedagogía para la Educación Superior. Universidad del Bío-Bío. Chillán, Chile. Email: aastudil@ubiobio.cl. Autor de correspondencia.

**Enfermera. Universidad del Bío-Bío Chillán, Chile. Email: psilvap@egresados.ubiobio.cl

***Enfermera. Hospital Regional de Antofagasta Dr. Leonardo Guzmán Chile. Email: jenidaza3112@gmail.com
} 
research is to determine the stress level in parents whose children have been hospitalized in neonatal and pediatric critical care units, and how this relates to the socio-demographic characteristics of the parent and/or child. Materials and Methods: Quantitative, descriptive correlational and cross-sectional study, using a sample of 41 suitable parents, who were present in May 2017 in the pediatric and neonatal critical care units of a hospital located in the Nuble Region, Chile. A characterization questionnaire and the Spanish version of the Parental Stress Scale adapted by Polaino-Lorente and Ochoa were applied. The data were processed using SPSS 15.0, performing Student's $t$ and one-way ANOVA tests. Results: According to the overall stress score, $48.8 \%$ classified the level as extremely stressful; $29.3 \%$ as very stressful; $12.2 \%$ as moderately stressful and the rest as little and not stressful at all. Stress in parents is higher during the child's first week of hospitalization, both in areas of communication with the health professionals and the alteration of the parental role. Conclusion: Stress in parents with a hospitalized child in critical care units can be extreme, being higher during the child's first week of hospitalization.

Key words: Psychological stress; Parents; Neonatal nursing; Pediatric, nursing; Intensive Care Units.

\section{RESUMO}

Introdução: A doença e a hospitalização são eventos críticos que enfrenta uma criança, onde a reação de estresse é comum em toda a família durante a doença ou uma internação grave. Objetivo: Determinar o nível de estresse em pais de crianças internadas em unidades críticas de pediatria e neonatologia e a relação com características sociodemográficas do pai e/ou a criança. Material e método: Estudo quantitativo, descritivo-correlacional, de corte transversal. A amostra estudada foi de 41 pais, que foram elegíveis e estiveram presentes em maio de 2017 nas unidades críticas de pediatria e neonatologia de um hospital localizado na Región del Nuble, Chile. Foi aplicado um instrumento de caracterização e a versão em espanhol da Escala de Estresse Parental adaptada por Polaino-Lorente e Ochoa. Os dados foram processados com SPSS 15.0, realizando testes t-student e ANOVA de um fator. Resultados: Em relação à pontuação global de estresse, 48,8\% classificou o grau de estresse como extremadamente estressante; $29,3 \%$ muito estressante; $12,2 \%$ moderadamente estressante e o restante pouco e nada estressante. $\mathrm{O}$ estresse em pais é maior na primeira semana de hospitalização da criança, nas áreas de comunicação do pessoal de saúde e a alteração do rol dos pais, sendo ambas significativas. Conclusão: $\mathrm{O}$ estresse em pais com um filho internado em unidades críticas resulta na maioria dos casos extremo e é ainda maior durante a primeira semana de hospitalização da criança.

Palavras-chave: Estresse Psicológico; Pais, Enfermagem Neonatal; Enfermagem Pediátrica; Unidades de Terapia Intensiva Neonatal.

Fecha recepción: 14/08/2018

Fecha aceptación: 13/05/2019

\section{INTRODUCCIÓN}

Desde hace muchos años se cree que durante las hospitalizaciones es fundamental curar la enfermedad sin importar el método, ni tener en cuenta las repercusiones psicológicas que las hospitalizaciones provocan en el paciente y su familia ${ }^{(1)}$.

La participación de los padres en las unidades críticas neonatales es sumamente escasa, con poco reconocimiento consciente por parte de ellos mismos, percibiendo esta limitación como algo normalizado, no empoderada, y no concibiéndose a sí mismos como sujetos con posibilidades de participación activa en el cuidado de sus hijos internados, por lo cual aumenta el sufrimiento, lo que no deja de ser importante en un proceso humanizado de cuidado(2).

La enfermedad y la hospitalización son a menudo eventos críticos a los que se enfrenta un niño y la reacción de estrés en toda la familia es común durante la hospitalización grave. De 
igual manera ocurre con el recién nacido ( $\mathrm{RN})$ prematuro, debido a que su situación de salud requiere cuidados especiales en unidad de cuidado intensivo neonatal (UCIN), con hospitalizaciones largas que pueden durar días e incluso meses y cuyas consecuencias pueden ser la disrupción de la relación padres-hijo, afectando su desarrollo físico y emocional $^{(3)}$.

Diversos estudios ${ }^{(4-7)}$ han hecho evidente la presencia de estrés en los padres durante la hospitalización de niños de todas las edades, y la importancia de la presencia y apego de los padres. Por ejemplo, Board y Ryan-Wenger de los Estados Unidos $^{(4)}$ reportaron que el $90 \%$ de las madres padecen estrés durante la hospitalización de sus hijos en la Unidad de Cuidados Intensivos (UCI) pediátrica.

En el estudio de Wormald et al. ${ }^{(5)}$, en el cual participaron 12 unidades de neonatología de cuatro países (Argentina, Chile, Paraguay, Perú) que forman parte de la Red Neocosur, se utilizó la versión validada al español de la escala de estrés parental, diseñada por Miles et al. ${ }^{(8)}$, mostrando que la alteración del rol parental era la situación que mayor estrés les provocaba a los padres.

En muchos casos, se plantea que la experiencia del ingreso a la UCI neonatal y pediátrica es más estresante para la familia que para el propio paciente, debido a que se ve obligada a descuidar sus necesidades básicas, originando, por ejemplo, menor descanso, alimentación inadecuada, abandono o exceso de trabajo ${ }^{(9)}$. Al mismo tiempo esto genera estrés y alteración en su estado de salud, denotándose que el paciente y sus familiares son seres sensibles y vulnerables y que requieren el mayor apoyo posible para enfrentar los cambios que genera esta nueva experiencia. Debido a esto, el paciente pediátrico necesita que el personal de salud cuente con experiencia y diferentes competencias para su cuidado, en especial de los profesionales de enfermería, por ser ellos con quienes más interactúa durante su hospitalización en la UCI. De igual forma, los profesionales de enfermería deben contar con competencias para brindar a los padres el mayor apoyo posible, para afrontar de la mejor manera las alteraciones físicas y emocionales que puedan presentar. En pediatría es necesario involucrar a la familia en el cuidado, ya que el trabajo en equipo es clave durante el proceso de recuperación de estos pacientes ${ }^{(9)}$.
El ser padre o madre resulta muchas veces estresante en sí mismo. Desde el embarazo se presentan diferentes niveles de estrés, debido a los cambios físicos y hormonales, los cuales aumentan con el nacimiento. Conciliar la vida laboral con el cuidado de los hijos y las preocupaciones, entre otras cosas, puede ser una fuente de estrés adicional. Si además le sumamos una hospitalización, mayor resulta este estrés ${ }^{(10)}$.

Esta investigación se realizó con el objetivo de determinar el nivel de estrés de los padres de niños hospitalizados en unidades críticas pediátricas y neonatales y su relación con características sociodemográficas del padre y/o niño/a.

\section{MATERIAL Y MÉTODO}

El presente estudio fue de tipo cuantitativo, descriptivo-correlacional, de corte transversal. La población de estudio estuvo conformada por 82 padres de nińos hospitalizados en unidades críticas pediátricas y neonatales de un hospital en Chile, durante el mes de mayo de 2017. La población se determinó de acuerdo al número de camas ocupadas en el periodo de duración del estudio. La muestra estuvo conformada por 41 personas presentes en la sala de espera del Centro de Responsabilidad de Pediatría, que fueron todos los sujetos encuestados que cumplieron con los criterios de elegibilidad del estudio: ser padre o madre de un niño hospitalizado con una estadía igual o superior a un día y ser mayor de 18 ańos al momento de realizar el estudio. Se excluyó a quienes eran analfabetos, padres que estuvieran cursando algún tipo de duelo durante los últimos 6 meses y padres que presentaran depresión $\mathrm{u}$ otra enfermedad de salud mental conocida, determinado a través de preguntas directas.

Las variables estudiadas fueron: a) variable principal: estrés y sus subescalas; b) variables sociodemográficas del padre o madre: sexo, edad, escolaridad, número dehijos, estado civil, ocupación, ingreso económico per cápita y procedencia geográfica; c) variables en relación al niño y su hospitalización actual: edad, hospitalizaciones previas, patologías previas, suficiencia del horario de visita e información recibida por parte del personal de enfermería.

La recolección de datos se realizó de manera posterior a la autorización del estudio por parte 
del Comité Ético Científico (CEC) de un hospital chileno, ubicado en la Región de Nuble. Todos los padres que participaron en el estudio dieron su consentimiento informado siguiendo las directrices del CEC, y como se refleja en los postulados éticos contenidos en la declaración de Helsinki, así como la Ley 19.628, la Ley 20.120 y en la Ley $20.584^{(11-13)}$ de la legislación chilena vigente. Los detalles sobre el consentimiento informado figuran en un documento que fue leído y firmado por ambas partes, participante y un miembro del equipo de investigación; explícitamente se señaló que la colaboración es voluntaria, pudiendo abandonar el estudio en el momento deseado y que los investigadores se comprometen a velar por la confidencialidad de los datos.

Los datos fueron recolectados a través de 2 cuestionarios aplicados por las investigadoras a los padres. El primero fue un cuestionario de caracterización de la muestra, creado por las autoras del estudio, con el objetivo de recolectar información sociodemográfica, así como de sus hijos y la hospitalización actual. El cuestionario cuenta de quince preguntas de respuestas abiertas y cerradas, referentes a sexo, edad, edad del hijo, escolaridad, número de hijos, estado civil, ocupación, ingreso económico per cápita, procedencia geográfica, hospitalizaciones previas del niño, patologías previas del niño, suficiencia del horario de visita, información recibida por parte del personal de enfermería, tipo de alimentación que recibe el niño y los días que lleva hospitalizado. El segundo, la Escala de Estrés Parental (Parental Stressor Scale), creada por Carter y Miles ${ }^{(14)}$. Esta escala es de carácter multidimensional, desarrollada para medir y evaluar el estrés relacionado con la permanencia de los padres en las unidades de cuidados intensivos pediátricos $^{(14)}$. Para efectos de la medición, esta escala define una experiencia estresante como una vivencia que causa ansiedad o tensión ${ }^{(8)}$.

La versión en español de la escala Parental Stressor Scale (PSS) ${ }^{(15)}$ fue traducida en España por Polaino-Lorente y Ochoa en 1992, realizándose un estudio preliminar de validación utilizando análisis factorial. El coeficiente alpha de Cronbach global del instrumento original es de $0,99^{(14)}$ y el obtenido por los autores de la adaptación alcanzó un valor de 0,92 . Las subescalas obtuvieron valores desde 0,78 a 0,97 . Los resultados de este estudio preliminar de validez, no recomendaron su uso hasta contar con datos más robustos ${ }^{(15)}$; sin embargo, las autoras del presente estudio consideran que esta escala era la más adecuada para la evaluación de las variables estudiadas, además de no existir a la fecha de realización de este estudio otra escala validada en Chile que se ajustara a estas. Se consideró también el idioma y características sociodemográficas similares de los individuos participantes de los estudios realizados en España.

La escala consta de 36 ítems, divididos en 6 subescalas o categorías de estrés parental, apariencia física del niño, tratamiento recibido, comunicación con el personal sanitario, conductas y respuestas emocionales del niño, comportamiento del personal sanitario, alteración de los roles de los padres/expectativas de seguimiento. Además, se adiciona un último ítem (No 37), que pretende evaluar el impacto general causado por la hospitalización pediátrica en los padres estudiados, el cual consiste en una puntuación de estrés parental que es independiente, ya que no forma parte de las categorías. Las opciones de respuesta a los ítems corresponden al formato Likert, con un espectro de respuesta de cinco opciones que oscila desde 1 "no estresante", 2 "un poco estresante", 3 "moderadamente estresante", 4 "muy estresante" y 5 "extremadamente estresante", entendiéndose que a menor puntaje menor estrés y a mayor puntaje mayor estrés. En el caso que los padres no hubiesen experimentado la situación a la cual se refieren los ítems, la opción de respuesta corresponde a 0 . La Escala ofrece dos formas de puntuación: la primera, global, constituyendo un solo índice donde se debe dividir la suma de todas las respuestas por el número de ítems. De esta forma se obtiene una expresión general de estrés en los padres como consecuencia de la hospitalización. El segundo, puntuando cada subescala por separado, donde se debe dividir la suma de los resultados obtenidos en cada subescala por el número de ítems. En ambas formas de puntuación se incluyen solo los ítems que han sido valorados del 1 al 5 para el cálculo, lo que compensa los ítems no experimentados por los padres.

El levantamiento de datos se realizó en la sala de espera del Centro de Responsabilidad de pediatría, en condiciones que aseguraron una buena iluminación, silencio y privacidad, evitando 
que una tercera persona esté presente en el proceso del trabajo de campo. El instrumento recolector de datos fue autoadministrado. El procedimiento se realizó en presencia de las investigadoras, quienes entregaron instrucciones de manera uniforme para todos los participantes y de manera individual, evitando juicios y sin influir en las respuestas o sugerir respuestas socialmente aceptadas. Todos los entrevistados fueron captados de manera previa o posterior al horario de visita establecido en el servicio.

Las variables cuantitativas fueron descritas con desviación media y media aritmética. Con el objetivo de comparar los días de hospitalización respecto al estrés parental, se aplicó prueba t-student y ANOVA de un factor, bajo el supuesto de normalidad. En caso de que el supuesto no se cumplía, se aplicó la prueba de Kruskall-Wallis. Se trabajó con un nivel de significación estadística de 0,05. La gestión de datos fue realizada con el paquete estadístico SPSS, versión 15.0 en español.

\section{RESULTADOS}

En relación a la muestra evaluada, las características sociodemográficas se presentan en la Tabla 1, encontrándose que el 51,2\% eran de sexo femenino; el 73,2\% informó una edad entre 20 y 34 años; el $43,9 \%$ fue clasificado dentro del quintil 1 y 2 ; el $46,3 \%$ presentaba estudios medios, seguido de un $34,1 \%$ con estudios superiores; el 70,7\% indicó tener un solo hijo; el 51,2\% indicó como ocupación el ser trabajador dependiente; el $68,3 \%$ procede de zona urbana y el $61 \%$ informó ser soltero.

Con respecto a la distribución de la muestra estudiada, según las características de sus hijos y la hospitalización actual (Tabla 2), el 58,5\% de los padres tenían hijos recién nacidos, $31,7 \%$ en edades correspondientes a lactante menor y 9,8\% preescolares; el $73,2 \%$ de los hijos no presentaban hospitalizaciones previas; el $78 \%$ no presentaba patologías previas, ya sean crónicas o agudas que requirieran atención de urgencia; en cuanto a la hospitalización actual, el $63,4 \%$ de los padres manifestaron que se sentían conformes con el horario de visita establecido por el servicio en las unidades críticas de pediatría y neonatal; el 85,4\% dijo que la información entregada por el personal médico y de enfermería era suficiente; el 90,2\% de los niños se alimentaba por vía enteral y el 65,9\% de los nińos se encontraba dentro de su primera semana de hospitalización.

Los resultados medios de las diferentes subescalas o categorías de la escala de estrés parental, según se aprecia en la Tabla 3, más elevados corresponden a la alteración del rol de padres/expectativas de seguimiento; tratamiento recibido y la comunicación con el personal sanitario. Respecto al puntaje global de estrés indicado por los padres, se destaca que el $48,8 \%$ de los participantes del estudio clasifican el impacto o grado de estrés que les causó la hospitalización de su hijo como extremadamente estresante; un $29,3 \%$ la clasificó como muy estresante, un 12,2\% como moderadamente estresante y un $9,7 \%$ como poco o nada estresante.

En cuanto a los puntajes medios de las categorías de la escala de estrés aplicada a los padres según el número de días de hospitalización de sus hijos (Tabla 4), las categorías comunicación del personal sanitario y alteración rol de padres/expectativas de seguimiento mostraron diferencias significativas con un p-value de 0,024 para el primero y 0,001 para el segundo.

\section{DISCUSIÓN}

Se puede identificar que, dentro de las características sociodemográficas del grupo de estudio, tanto hombres como mujeres se encontraban en porcentajes muy similares, lo que contrasta con el estudio realizado por López ${ }^{(16)}$, donde se observó una gran brecha entre hombres y mujeres que correspondían a 29,4 y 70,6\%, respectivamente. Igualmente ocurrió en el estudio de Wormald et al.(5), donde un $27,5 \%$ de los padres eran hombres y un $72,5 \%$ eran mujeres. Esto puede explicarse por las realidades culturales de cada nación, y donde nuestro país muestra un cambio a lo largo de los años en cuanto a la manera de ver la crianza de los hijos y el rol de cada padre en su cuidado de estos. 
Tabla 1. Distribución de los padres participantes del estudio según sus características sociodemográficas.

\begin{tabular}{|c|c|c|}
\hline Características sociodemográficas & $\mathbf{N}$ & $\%$ \\
\hline \multicolumn{3}{|l|}{ Sexo } \\
\hline Hombre & 20 & 48,8 \\
\hline Mujer & 21 & 51,2 \\
\hline \multicolumn{3}{|l|}{ Edad del padre o madre (ańos) } \\
\hline$<20$ & 1 & 2,4 \\
\hline $20-34$ & 30 & 73,2 \\
\hline $35-49$ & 10 & 24,4 \\
\hline $50-64$ & 0 & - \\
\hline \multicolumn{3}{|c|}{ Ingreso económico (ingreso per cápita) } \\
\hline$\$ 0$ a $\$ 74.969$ & 8 & 19,5 \\
\hline$\$ 74.970$ a $\$ 125.558$ & 10 & 24,4 \\
\hline$\$ 125.559$ a $\$ 193.104$ & 8 & 19,5 \\
\hline$\$ 193.105$ a $\$ 352.743$ & 7 & 17,1 \\
\hline$\geq \$ 352.743$ & 8 & 19,5 \\
\hline \multicolumn{3}{|c|}{ Escolaridad (mayor nivel de estudios alcanzado) } \\
\hline Sin estudios & 2 & 4,9 \\
\hline Estudios básicos & 6 & 14,6 \\
\hline Estudios medios & 19 & 46,3 \\
\hline Estudios superiores & 14 & 34,1 \\
\hline \multicolumn{3}{|l|}{ Número de hijos } \\
\hline 1 & 29 & 70,7 \\
\hline 2 & 9 & 22 \\
\hline$\geq 3$ & 3 & 7,3 \\
\hline \multicolumn{3}{|l|}{ Ocupación } \\
\hline Trabajador independiente & 5 & 12,2 \\
\hline Trabajador dependiente & 21 & 51,2 \\
\hline Dueña de casa & 9 & 22 \\
\hline Estudiante & 3 & 7,3 \\
\hline Cesante & 3 & 7,3 \\
\hline \multicolumn{3}{|l|}{ Procedencia } \\
\hline Urbana & 28 & 68,3 \\
\hline Rural & 13 & 31,7 \\
\hline \multicolumn{3}{|l|}{ Estado civil } \\
\hline Soltero(a) & 25 & 61,0 \\
\hline Casado(a) & 13 & 31,7 \\
\hline Viudo(a) & 0 & - \\
\hline Divorciado(a) & 1 & 2,4 \\
\hline Conviviente civil & 2 & 4,9 \\
\hline
\end{tabular}

$1 \mathrm{USD}=794$ pesos chilenos. 
Tabla 2. Distribución de los padres participantes del estudio según características de sus hijos y la hospitalización actual.

\begin{tabular}{|c|c|c|}
\hline Características del nińo & $\mathbf{N}$ & $\%$ \\
\hline \multicolumn{3}{|l|}{ Edad del hijo (días, meses, años) } \\
\hline$\leq 29$ días & 24 & 58,5 \\
\hline 29 días a 11 meses 29 días & 13 & 31,7 \\
\hline 12 meses a 23 meses 29 días & 0 & - \\
\hline 2 años a 5 años 11 meses 29 días & 4 & 9,8 \\
\hline 6 ańos a 14 años 11 meses 29 días & 0 & - \\
\hline \multicolumn{3}{|l|}{ Hospitalizaciones previas del niño } \\
\hline 0 & 30 & 73,2 \\
\hline 1 & 3 & 7,3 \\
\hline 2 & 5 & 12,2 \\
\hline$\geq 3$ & 3 & 7,3 \\
\hline \multicolumn{3}{|l|}{ Patologías previas del niño } \\
\hline Sí & 9 & 22 \\
\hline No & 32 & 78 \\
\hline \multicolumn{3}{|l|}{ Horario de visita } \\
\hline Suficiente & 26 & 63,4 \\
\hline Insuficiente & 15 & 36,6 \\
\hline \multicolumn{3}{|l|}{ Información recibida } \\
\hline Suficiente & 35 & 85,4 \\
\hline Insuficiente & 6 & 14,6 \\
\hline \multicolumn{3}{|l|}{ Tipo de Alimentación } \\
\hline Enteral & 37 & 90,2 \\
\hline Parenteral & 4 & 9,8 \\
\hline \multicolumn{3}{|l|}{ Días de hospitalización } \\
\hline $1-3$ & 12 & 29,3 \\
\hline $4-7$ & 15 & 36,6 \\
\hline $8-11$ & 5 & 12,2 \\
\hline $12-15$ & 2 & 4,9 \\
\hline$\geq 16$ & 7 & 17,1 \\
\hline
\end{tabular}

Tabla 3. Medidas de resumen del puntaje que evalúa estrés parental según subescala.

\begin{tabular}{ll}
\hline Subescala & Nivel de estrés \\
\cline { 2 - 2 } & Media (DT) \\
\hline Apariencia física del niño & $2,88(1,107)$ \\
Tratamiento recibido & $3,20(1,112)$ \\
Comunicación con el personal sanitario & $3,03(0,950)$ \\
Conductas y respuestas emocionales del niño & $2,74(1,215)$ \\
Comportamiento del personal sanitario & $2,72(1,317)$ \\
Alteración roles de padres/ expectativas de seguimiento & $3,38(1,241)$ \\
\hline Puntaje global & $4,15(1,062)$ \\
\hline
\end{tabular}


Tabla 4. Medidas de resumen del puntaje que evalúa estrés parental según número de días de hospitalización.

\begin{tabular}{lcccc}
\hline \multirow{2}{*}{ Nivel de estrés } & $\begin{array}{c}\mathbf{7} \text { días }(\mathbf{n}=\mathbf{2 7}) \\
\text { Media }(\mathbf{D T})\end{array}$ & $\begin{array}{c}\mathbf{>} \text { días }(\mathbf{n}=\mathbf{1 4}) \\
\mathbf{M e d i a}(\mathbf{D T})\end{array}$ & $\mathbf{t}$ & p-valor \\
\hline Apariencia física del nińo & $3,02(1,139)$ & $2,61(1,034)$ & 1,13 & $0.2668^{*}$ \\
Tratamiento recibido & $3,33(1,117)$ & $2,95(1,100)$ & 1,04 & $0.3056^{*}$ \\
Comunicación con el personal sanitario & $3,30(0,985)$ & $2,60(0,727)$ & 2,34 & $\mathbf{0 . 0 2 4 3}^{*}$ \\
Conductas y respuestas emocionales del niño & $2,81(1,388)$ & $2,62(0,916)$ & 0,46 & $0.6030^{* *}$ \\
Comportamiento del personal sanitario & $2,95(1,371)$ & $2,36(1,178)$ & 1,37 & $0.1792^{*}$ \\
Alteración roles de padres/ expectativas de seguimiento & $3,83(1,056)$ & $2,54(1,145)$ & 3,61 & $\mathbf{0 . 0 0 0 9}^{*}$ \\
\hline Puntaje global & $4,22(1,121)$ & $4,00(0,961)$ & 0,62 & $0.5362^{*}$ \\
\hline
\end{tabular}

*Prueba t-Student, una vez asumida homogeneidad de varianzas.

**Prueba t-Student ajustada a heterogeneidad de varianzas.

En cuanto a la edad, el promedio fue de 28,78 años, concentrándose mayormente en el rango de adulto joven, datos que se asemejan a los encontrados por Rojas Carrasco ${ }^{(17)}$, donde se obtuvo que el $40 \%$ de las madres tenían edades entre 30 a 39 años y en el caso de los padres, el $46 \%$ fluctuaban entre 20 a 29 años. Otro estudio que respalda los datos obtenidos en la presente investigación es el de Díaz ${ }^{1}$, quien evidenció que el rango de edad fue entre 18 y 55 años, con un promedio de 28,71 años. La semejanza de los rangos etarios que priman en cada estudio y el promedio de estos puede deberse a la similitud en general que tienen los países latinoamericanos en cuanto a su nivel de desarrollo y al proceso de maternidad postergado por la mujer para priorizar su desempeño profesional y laboral.

En el nivel socioeconómico se observó una prevalencia de estrato bajo y medio, concordando con Giraldo et al. ${ }^{(18)}$, donde el $42 \%$ pertenecía a un nivel económico medio bajo, y López ${ }^{(16)}$, donde la mayoría correspondió a un nivel económico medio.

En cuanto a la escolaridad, predominaron los padres con estudios medios, seguidos de padres con estudios superiores, encontrándose pocos casos con estudios básicos o sin escolaridad. López ${ }^{(16)}$ observó que, a mayor nivel de formación académica, menor era el nivel de estrés. Wormald et al. ${ }^{(5)}$ también encontraron que las características que se asociaron a un mayor nivel de estrés parental fueron tener un nivel educacional bajo. Se puede presumir que el factor protector de escolaridad elevada puede haber significado un menor nivel de estrés en los padres. Con respecto al número de hijos, la mayoría de los padres encuestados eran padres primerizos. El estado civil predominante fue soltero, lo que refleja la situación país actual.

En cuanto a la situación de ocupación de los sujetos, se observó que más de la mitad de ellos se desempeñaba como trabajador dependiente, tal como ocurrió con Giraldo et al. ${ }^{(18)}$, con un $62 \%$ de empleados dependientes y Aguinaga-Zamarripa et al. ${ }^{(19)}$, con un $51 \%$ de empleados dependientes.

En relación a la procedencia geográfica, casi dos tercios correspondía a urbana y un tercio a rural, destacándose el alto porcentaje de ruralidad en comparación con el promedio comunal y regional, de 8,6 y $24,2 \%$, respectivamente ${ }^{(20)}$.

Por otra parte, según las características de los hijos y la hospitalización actual, el mayor porcentaje de niños correspondían a recién nacidos (<29 días) que estaba hospitalizado por primera vez y no presentaban ninguna patología previa, lo que se respalda con la mayoría de las investigaciones mencionadas anteriormente, tales como, Giraldo et al. ${ }^{(18)}$, Aguiñaga-Zamarripa et al. ${ }^{(19)}$, Díaz ${ }^{1}$ y González et al. ${ }^{(21)}$.

La apreciación de los padres respecto del horario de visita establecido en el servicio fue suficiente en el $63,4 \%$ de los encuestados. Esto puede deberse

${ }^{1}$ Díaz C. Participación ocupacional y estrés en padres de recién nacidos internados en unidades neonatales [Tesis de grado]. Universidad abierta Interamericana; 2012. 
a que los padres están conscientes del horario restringido de las unidades de cuidados críticos pediátricos y neonatales del hospital. Respecto a la información recibida por parte del personal médico y el equipo de enfermería, los padres la catalogaron en su mayoría como suficiente. Santos et al. ${ }^{(22)}$ indican que "la comunicación puede ser un recurso terapéutico o nocivo, en el contexto de la asistencia sanitaria. De esta manera, ella puede ser usada para beneficiar a la familia del niño hospitalizado y ayudarla en la vivencia/superación de momentos difíciles o puede comprometer aún más su salud física y mental, proporcionando momentos traumáticos y desestructurantes", por lo tanto, en el quehacer enfermero es muy importante poner énfasis en la comunicación con los familiares de nuestros usuarios, prestándoles atención, dando explicaciones claras y evitando el uso de celulares y otros elementos distractores durante la atención y entrega de información al paciente y su familia.

Al momento de realizar la encuesta la mayoría de los niños se encontraban entre el día 1 al 7 de su hospitalización, lo que implica cortas estadías hospitalarias que se pueden atribuir a las causas de ingreso de los pacientes, que en su mayoría se debieron a bajo peso al nacer o patologías propias del $\mathrm{RN}$ prematuro, condiciones que evolucionaban favorablemente, y permitían trasladar a los nińos a unidades básicas, no generando largos periodos de internación hospitalaria en las unidades críticas.

Refiriéndose al número de días de hospitalización, se obtuvo una diferencia significativa en las subescalas de comunicación con el personal sanitario y la alteración del rol de padres, siendo mayor el estrés durante los primeros 7 días de hospitalización. Este estrés parental puede verse disminuido realizando intervenciones tales como, explicaciones claras, fomentar el realizar preguntas al médico de las dudas que puedan tener respecto al diagnóstico de sus hijos, o ayudar a los padres a encontrar tareas que puedan realizar para contribuir con el cuidado de sus hijos. Esta última actividad favorecería disminuir el estrés dado por la segunda categoría, alteración de los roles de padres / expectativas de seguimiento. En dicha subescala los padres opinan sobre el grado de nerviosismo o inquietud que le provocan determinados cambios en su forma de vivir, ocasionados por la necesidad de atender las nuevas necesidades de un hijo enfermo y hospitalizado, donde se presentan preguntas referentes a la dificultad del cuidado y contacto con su hijo. Lo expuesto concuerda con Wormald et al. ${ }^{(5)}$, donde el ítem que puntuó más alto fue estar separado de su hijo. Lecannelier et al. ${ }^{(23)}$ sostiene que "De acuerdo a la evidencia empírica, el desarrollo normal de un nińo requiere de una relación cálida, íntima y continua con la madre", es por ello que resulta imperioso fomentar el apego padres hijos y respaldar la labor de padres que estos puedan tener en la unidad crítica donde se encuentre su hijo.

En cuanto al puntaje global de la escala, se observó que muy cercano a la mitad de los participantes de la presente investigación clasificó la experiencia de hospitalización de su hijo como extremadamente estresante. Dichos resultados son considerablemente superiores a los obtenidos por cada subescala, lo que pudo deberse a que la pregunta planteada en esta parte de la escala de estrés parental "¿Cuál es el impacto o grado de estrés que le ha causado la hospitalización de su hijo?” les resultaba una manera de expresar más concretamente lo mucho que les agobiaba esta situación. Esto se equipara con González et al. ${ }^{(21)}$, quienes obtuvieron que el $89 \%$ de las madres de nińos en la Unidad de Cuidados Intensivos Neonatales indicaron que la situación fue muy o extremadamente estresante, en este caso ambas categorías alcanzan el 78,1\% del total. López ${ }^{(16)}$ también obtuvo que el 69,4\% de los padres refirió que la situación era muy o extremadamente estresante.

\section{CONCLUSIONES}

De manera global, y de acuerdo a la percepción de los padres, se concluye que el estrés en padres con un hijo hospitalizado en unidades críticas resulta en la mayoría de los casos extremo, independiente de las características sociodemográficas de estos.

De acuerdo al análisis de los datos recolectados durante la investigación, podemos determinar que, para los padres, el no poder ejercer su rol es la situación que mayor estrés les provoca, destacándose por sobre todas las demás categorías evaluadas.

En cuanto al nivel de estrés y el número de días de hospitalización, se pudo determinar que quienes se encontraban dentro de la primera semana de hospitalización padecían de mayores niveles de estrés, destacándose en las subescalas de comunicación con el personal sanitario y su 
alteración del rol de padres, ambas significativas, por lo que podemos concluir que durante los primeros días de hospitalización los padres están más estresados y esto baja al prolongarse la estancia hospitalaria.

\section{REFERENCIAS}

1. Méndez F, Ortigosa J, Pedroche S. Preparación a la hospitalización infantil (I): Afrontamiento del estrés. Psicol Conductual. 1996; 4(2): 193-209.

2. Gallegos-Martínez J, Reyes-Hernández J, Scochi CG. El neonato prematuro hospitalizado: significado para los padres de su participación en la Unidad Neonatal. Rev Lat Am Enfermagem. [Internet]. 2013 Nov-Dic [citado 28 Jun 2018]; 21(6): 1360-66. Disponible en: http://dx.doi. org/10.1590/0104-1169.2970.2375

3. Fernández Castillo A, López Naranjo I. Estrategias para abordar el estrés de padres/madres e hijos/as en la hospitalización infantil: Dédalo [Internet]. Sevilla: Junta de Andalucía Consejería de Salud; 2012 Sep 7 [citado 5 May 2018]; [alrededor 1 pantalla]. Disponible en: https://reddedalo. wordpress.com/2012/09/07/estrategias-paraabordar-el-estres-de-padresmadres-e-hijosas-en-lahospitalizacion-infantil/

4. Board R, Ryan-Wenger N. Long-term effects of pediatric intensive care unit hospitalization on families with young children. Heart Lung. [Internet]. 2002 Ene-Feb [citado 28 Jun 2018]; 31(1):53-66. Disponible en: https://www.ncbi. nlm.nih.gov/pubmed/11805751

5. Wormald F, Tapia JL, Torres G, Cánepa P, González MA, Rodríguez D, et al. Estrés en padres de recién nacidos prematuros de muy bajo peso hospitalizados en unidades de cuidados intensivos neonatales. Arch Argent Pediatr. [Internet]. 2015 [citado 28 Jul 2018]; 113(4):303-309. Disponible en: https://www.sap.org.ar/docs/publicaciones/ archivosarg/2015/v113n4a04.pdf

6. Ruiz AL, Ceriani JM, Cravedi V, Rodríguez D. Estrés y depresión en madres de prematuros: un programa de intervención. Arch Argent Pediatr. 2005 103(1):36-45.

7. Ramírez M, Pino P, Springmuller D, Clavería C. Estrés en padres de niños operados de cardiopatías congénitas. Arch Argent Pediatr. 2014; 112(3):263-67.

8. Miles MS, Funk SG, Carlson J. Parental Stressor Scale: neonatal intensive care unit. Nurs Res. 1993; 42(3):148-152.

9. Alvarado Romero HR, Rodríguez Colmenares SM.

\section{Limitaciones del estudio:}

Utilización de la Escala de estrés parental aún no validada para su traducción al español y para el contexto chileno.
Necesidades percibidas de atención por niños, cuidadores y enfermeros durante la hospitalización en una unidad de cuidado intensivo. Investig. Enferm. Imagen Desarr. [Internet]. 2015 Ene-Jun [citado 28 Jul 2018]; 17(1): 113-130. Disponible en: https://revistas.javeriana.edu.co/index.php/ imagenydesarrollo/article/view/8665

10. Muñoz A. El estrés en los padres [Internet]. Málaga: Centro de Psicología Virtual; [citado 14 Nov 2019]. Disponible en: https://www.cepvi. com/index.php/psicologia-infantil/articulos/elestres-en-los-padres

11. Ministerio Secretaría General de la Presidencia Chile. Ley $\mathrm{N}^{\circ} 19.628$ Sobre protección de la vida privada, 18 Ago 1999 [Internet]. Valparaíso, Chile: Biblioteca del Congreso Nacional de Chile; 1999 [citado 28 Jul 2018]. Disponible en: https://www.leychile.cl/ Navegar?idNorma $=141599$ \&buscar $=19628$

12. Ministerio de Salud Chile, Subsecretaría de Salud Pública. Ley $\mathrm{N}^{\circ}$ 20.120. Sobre la Investigación Científica en el Ser Humano, su Genoma y Prohíbe la Clonación Humana, 07 Sept 2006 [Internet]. Valparaíso, Chile: Biblioteca del Congreso Nacional de Chile; 2006 [citado 28 May 2018]. Disponible en: https://www.leychile.cl/ Navegar $?$ idNorma $=253478 \&$ buscar $=20120$

13. Ministerio de Salud Chile, Subsecretaría de Salud Pública. Ley $\mathrm{N}^{\circ}$ 20.584. Regula los derechos y deberes que tienen las personas en relación con acciones vinculadas a su atención en salud, 06 Jun 2015 [Internet]. Valparaíso, Chile: Biblioteca del Congreso Nacional de Chile. [citado 28 Mayo 2018]. Disponible en: https://www.leychile.cl/ Navegar? idNorma $=1039348 \&$ buscar $=20584$

14. Carter MC, Miles MS. The parental stressor scale: pediatric intensive care unit. Matern Child Nurse J. 1989; 18(3):187-98.

15. Ochoa B, Reparaz Ch, Polaino-Lorente A. Estudios preliminares de validez de la escala PSS: Cuestionario de estrés en padres de niños hospitalizados. Clin Salud. 1997; 8(3): 423-446.

16. López I. Alteraciones emocionales en la hospitalización infantil: análisis psicoevolutivo. [Tesis Doctoral]. Granada: Editorial de la Universidad de Granada; 2011. 285 p.

17. Rojas Carrasco KE. Niveles de ansiedad en madres y padres con un hijo en terapia intensiva. Rev Med 
Inst Mex Seguro Soc [Internet]. 2013 [citado 28 Jun 2018]; 51(6): 606-609. Disponible en: https:// www.medigraphic.com/cgi-bin/new/resumen. cgi?IDARTICULO=46593

18. Giraldo D, Lopera F, Duque Y, Londoño A, Gaviria C, Restrepo A et al. Estrés y factores relacionados en padres con hijos en la unidad de cuidado intensivo neonatal. Horiz Enferm. [Internet]. 2012 [citado 28 Jun 2018]; 23(3): 13-22. Disponible en: http://horizonteenfermeria.uc.cl/images/pdf/23-3/ estres_factores.pdf

19. Aguiñaga-Zamarripa M, Reynaga-Ornelas L, Beltrán-Torres A. Estrés percibido por los padres del neonato en estado crítico durante el proceso de hospitalización. Rev Enferm Inst Mex Seguro Soc. [Internet]. 2016 [citado 28 Jun 2018]; 24(1): 2735. Disponible en: https://www.medigraphic.com/ pdfs/enfermeriaimss/eim-2016/eim161f.pdf

20. Biblioteca del Congreso Nacional/BCN. [Internet]. Chile: Regiones, Chile Nuestro País. Indicadores sociodemográficos y económicos Región del Biobío. 2017 [citado 28 Jul 2018]; Disponible en: http:// www.bcn.cl/siit/nuestropais/region8/indica.htm
21. González Escobar D, Ballesteros Celis N, Serrano Reatiga M. Determinantes estresores presentes en madres de neonatos pretérmino hospitalizados en las Unidades de Cuidados Intensivos. Rev Ciencia y Cuidado. [Internet]. 2012 Jul [citado 28 Jun 2018]; 9(1):43-53. Disponible en: https://revistas. ufps.edu.co/index.php/cienciaycuidado/article/ view/455

22. Santos LF, Oliveira LMAC, Munari DB, Barbosa MA, Peixoto MKAV, Nogueira ALG. Cuando la comunicación es nociva en el encuentro entre profesional y familia del niño hospitalizado. Enferm. glob. [Internet]. 2015 [citado 28 Jun 2018]; 14(37): 192-203. Disponible en: http:// scielo.isciii.es/pdf/eg/v14n37/docencia4.pdf

23. Lecannelier F, Undurraga V, Olivares AM, Rodríguez J, Núñez JC, Hoffmann M, et al. Estudio de eficacia sobre dos intervenciones basadas en el fomento del apego temprano en díadas madrebebé provenientes de la Región Metropolitana en Santiago de Chile. Revista Argentina de Clínica Psicológica. 2009; 18 (2):143-55. 